\title{
Monotone Data Visualization Using Rational Trigonometric Spline Interpolation
}

\author{
Farheen Ibraheem, ${ }^{1}$ Maria Hussain, ${ }^{2}$ and Malik Zawwar Hussain ${ }^{3}$ \\ ${ }^{1}$ National University of Computer and Emerging Sciences, Lahore, Pakistan \\ ${ }^{2}$ Department of Mathematics, Lahore College for Women University, Lahore 54600, Pakistan \\ ${ }^{3}$ Department of Mathematics, University of the Punjab, Lahore 54590, Pakistan
}

Correspondence should be addressed to Maria Hussain; mariahussain_1@yahoo.com

Received 3 January 2014; Accepted 5 February 2014; Published 3 April 2014

Academic Editors: A. Bellouquid and A. A. Elsadany

Copyright (C) 2014 Farheen Ibraheem et al. This is an open access article distributed under the Creative Commons Attribution License, which permits unrestricted use, distribution, and reproduction in any medium, provided the original work is properly cited.

\begin{abstract}
Rational cubic and bicubic trigonometric schemes are developed to conserve monotonicity of curve and surface data, respectively. The rational cubic function has four parameters in each subinterval, while the rational bicubic partially blended function has eight parameters in each rectangular patch. The monotonicity of curve and surface data is retained by developing constraints on some of these parameters in description of rational cubic and bicubic trigonometric functions. The remaining parameters are kept free to modify the shape of curve and surface if required. The developed algorithm is verified mathematically and demonstrated graphically.
\end{abstract}

\section{Introduction}

The technique or algorithm employed in creating images, diagrams, or animations for imparting a piece of information is termed as visualization. It has a key role to play in different fields like science, engineering, education, and medicine as it can aid experts in identifying and interpreting different patterns and artifacts in their data and provide a threedimensional display of data for the solution of a wide range of problems.

The methods used to obtain visual representations from abstract data have been in practice for a long time. However, physical quantities often emanate distinctive features (such as positivity, convexity, and monotonicity) and it becomes imperative that the visual model must contain the shape feature to fathom the physical phenomenon, the scientific experiment, and the idea of the designer. Spline interpolating functions play elemental role in visualizing shaped data. This paper specifically addresses the problem of visualizing monotone curve and surface data.

Monotonicity is an indispensable characteristic of data stemming from many physical and scientific experiments. The relationship between the partial pressure of oxygen and percentage dissociation of hemoglobin, consumption function in economics, concentration of atrazine and nitrate in shallow ground waters, and approximation of couples and quasi couples are few phenomena which exhibit monotone trend.

Efforts have been put in by many researchers and a variety of approaches has been proposed to solve this eminent issue [1-17]. Cripps and Hussain [3] visualized the 2D monotone data by Bernstein-Bézier rational cubic function. The authors in [3] converted the Bernstein-Bézier rational cubic function to $C^{1}$ cubic Hermite by applying the $C^{1}$ continuity conditions at the end points of interval. The lower bounds of weights functions were determined to visualize monotone data as monotone curve. Hussain and Sarfraz [8] have conserved monotonicity of curve data by rational cubic function with four shape parameters, two of which were set free and two were shape parameters. Data dependent constraints on shape parameters were developed which assure the monotonicity but one shape parameter is dependent on the other which makes it economically very expensive. Rational cubic function with two shape parameters suggested by Sarfraz [13] sustained monotonicity of curves but lacked the liberty to amend the curve which makes it inappropriate for interactive design. Piecewise rational cubic function was used by $\mathrm{M}$. 
Z. Hussain and M. Hussain [7] to visualize 2D monotone data by developing constraints on the free parameters in the specification of rational cubic function. The authors also extended rational cubic function to rational bicubic partially blended function. Simple constraints were derived on the free parameters in the description of rational bicubic partially blended patches to visualize the 3D monotone data. Three kinds of monotonicity preservation of systems of bivariate functions on triangle were defined and studied by Floater and Peña [5]. Sarfraz et al. [12] developed constraints in the specification of a bicubic function to visualize the shape of $3 \mathrm{D}$ monotone data.

This paper is a noteworthy addition in the field of shape preservation when the data under consideration admits monotone trend. The suggested algorithm offers numerous advantages over the prevailing ones. Orthogonality of sine and cosine function compels much smoother visual results as compared to algebraic spline. Derivative of the trigonometric spline is much lower than that of algebraic spline. Moreover, trigonometric splines play an instrumental role in robotic manipulator path planning.

The remainder of the paper is structured as follows. Section 2 is devoted to reviewing the rational trigonometric cubic function developed in [11]. In Section 3, rational trigonometric cubic function is extended to rational trigonometric bicubic function. Section 4 aims to develop monotonicity preserving constraints for 2D data. Section 5 submits a solution to shape preservation of $3 \mathrm{D}$ monotone data. In Section 6, numerical examples have been demonstrated. Section 7 draws the conclusion and significance of this research.

\section{Rational Trigonometric Cubic Function}

In this section, rational trigonometric cubic function [11] is reviewed.

Let $\left\{\left(x_{i}, f_{i}\right), i=0,1,2, \ldots, n\right\}$ be the given set of data points defined over the interval $[a, b]$, where $a=x_{0}<x_{1}<$ $x_{2}<\cdots<x_{n}=b$. Piecewise rational trigonometric cubic function is defined over each subinterval $I_{i}=\left[x_{i}, x_{i+1}\right]$ as

$$
\begin{gathered}
S_{i}(x)=\frac{p_{i}(\theta)}{q_{i}(\theta)}, \\
p_{i}(\theta)=\alpha_{i} f_{i}(1-\sin \theta)^{3}+\left\{\beta_{i} f_{i}+\frac{2 h_{i} \alpha_{i} d_{i}}{\pi}\right\} \\
\times \sin \theta(1-\sin \theta)^{2}+\left\{\gamma_{i} f_{i+1}-\frac{2 h_{i} \delta_{i} d_{i+1}}{\pi}\right\} \\
\times \cos \theta(1-\cos \theta)^{2}+\delta_{i} f_{i+1}(1-\cos \theta)^{3}, \\
q_{i}(\theta)=\alpha_{i}(1-\sin \theta)^{3}+\beta_{i} \sin \theta(1-\sin \theta)^{2} \\
+\gamma_{i} \cos \theta(1-\cos \theta)^{2}+\delta_{i}(1-\cos \theta)^{3},
\end{gathered}
$$

where $\theta=(\pi / 2)\left(\left(x-x_{i}\right) / h_{i}\right), h_{i}=x_{i+1}-x_{i}$.
The rational trigonometric cubic function (1) is $C^{1}$; that is, it satisfies the following properties:

$$
\begin{array}{ll}
S\left(x_{i}\right)=f_{i}, & S\left(x_{i+1}\right)=f_{i+1}, \\
S^{\prime}\left(x_{i}\right)=d_{i}, & S^{\prime}\left(x_{i+1}\right)=d_{i+1} .
\end{array}
$$

Here $d_{i}$ and $d_{i+1}$ are derivatives at the end points of the interval $I_{i}=\left[x_{i}, x_{i+1}\right]$. The parameters $\alpha_{i}$ and $\delta_{i}$ are real numbers used to modify the shape of the curve.

\section{Rational Trigonometric Bicubic Partially Blended Function}

Let $\left\{\left(x_{i}, y_{j}, F_{i, j}\right), i=0,1,2, \ldots, n-1 ; j=0,1,2, \ldots, m-1\right\}$ be the $3 \mathrm{D}$ regular data set defined over the rectangular mesh $I=$ $[a, b] \times[c, d]$, let $p: a=x_{0}<x_{1}<\cdots<x_{m}=b$ be a partition of $[a, b]$, and let $q: a=y_{0}<y_{1}<\cdots<y_{n}$ be a partition of $[c, d]$. Rational trigonometric bicubic function which is an extension of rational trigonometric cubic function (1) is defined over each rectangular patch $\left[x_{i}, x_{i+1}\right] \times\left[y_{j}, y_{j+1}\right]$, where $i=0,1,2, \ldots, n-1 ; j=0,1,2, \ldots, m-1$, as

$$
S(x, y)=-A F B^{T}
$$

where

$$
\begin{aligned}
& F=\left(\begin{array}{ccc}
0 & S\left(x, y_{j}\right) & S\left(x, y_{j+1}\right) \\
S\left(x_{i}, y\right) & S\left(x_{i}, y_{j}\right) & S\left(x_{i}, y_{j+1}\right) \\
S\left(x_{i+1}, y\right) & S\left(x_{i+1}, y_{j}\right) & S\left(x_{i+1}, y_{j+1}\right)
\end{array}\right) \\
& A=\left[\begin{array}{lll}
-1 & a_{0}(\theta) & a_{1}(\theta)
\end{array}\right], \quad B=\left[\begin{array}{lll}
-1 & b_{0}(\theta) & b_{1}(\theta)
\end{array}\right], \\
& a_{0}=\cos ^{2} \theta, \quad a_{1}=\sin ^{2} \theta, \\
& b_{0}=\cos ^{2} \varphi, \quad b_{1}=\sin ^{2} \varphi .
\end{aligned}
$$

$S\left(x, y_{j}\right), S\left(x, y_{j+1}\right), S\left(x_{i}, y\right)$, and $S\left(x_{i+1}, y\right)$ are rational trigonometric bicubic functions defined on the boundary of rectangular patch $\left[x_{i}, x_{i+1}\right] \times\left[y_{j}, y_{j+1}\right]$ as

$$
\begin{aligned}
S\left(x, y_{j}\right)= & \left(A_{0}(1-\sin \theta)^{3}+A_{1} \sin \theta(1-\sin \theta)^{2}\right. \\
& \left.+A_{2} \cos \theta(1-\cos \theta)^{2}+A_{3}(1-\cos \theta)^{3}\right) \\
& \times\left(q_{1}(\theta)\right)^{-1},
\end{aligned}
$$

where

$$
\begin{gathered}
A_{0}=\alpha_{i, j} F_{i, j}, \quad A_{1}=\beta_{i, j} F_{i, j}+\frac{2 \alpha_{i, j} h_{i} F_{i, j}^{x}}{\pi}, \\
A_{2}=\gamma_{i, j} F_{i+1, j}-\frac{2 \delta_{i, j} h_{i} F_{i+1, j}^{x}}{\pi}, \quad A_{3}=\delta_{i, j} F_{i+1, j}, \\
q_{1}(\theta)=\alpha_{i, j}(1-\sin \theta)^{3}+\beta_{i, j} \sin \theta(1-\sin \theta)^{2} \\
+\gamma_{i, j} \cos \theta(1-\cos \theta)^{2}+\delta_{i, j}(1-\cos \theta)^{3},
\end{gathered}
$$




$$
\begin{aligned}
S\left(x, y_{j+1}\right)= & \left(B_{0}(1-\sin \theta)^{3}+B_{1} \sin \theta(1-\sin \theta)^{2}\right. \\
& \left.+B_{2} \cos \theta(1-\cos \theta)^{2}+B_{3}(1-\cos \theta)^{3}\right) \\
& \times\left(q_{2}(\theta)\right)^{-1},
\end{aligned}
$$

where

$$
\begin{gathered}
B_{0}=\alpha_{i, j+1} F_{i, j+1}, \quad B_{1}=\beta_{i, j+1} F_{i, j+1}+\frac{2 \alpha_{i, j+1} h_{i} F_{i, j+1}^{x}}{\pi}, \\
B_{2}=\gamma_{i, j+1} F_{i+1, j+1}-\frac{2 \delta_{i, j+1} h_{i} F_{i+1, j+1}^{x}}{\pi}, \quad B_{3}=\delta_{i, j+1} F_{i+1, j+1}, \\
q_{2}(\theta)=\alpha_{i, j+1}(1-\sin \theta)^{3}+\beta_{i, j+1} \sin \theta(1-\sin \theta)^{2} \\
+\gamma_{i, j+1} \cos \theta(1-\cos \theta)^{2}+\delta_{i, j+1}(1-\cos \theta)^{3} \\
S\left(x_{i}, y\right)=\left(C_{0}(1-\sin \varphi)^{3}+C_{1} \sin \varphi(1-\sin \varphi)^{2}\right. \\
\left.+C_{2} \cos \varphi(1-\cos \varphi)^{2}+C_{3}(1-\cos \varphi)^{3}\right) \\
\times\left(q_{3}(\varphi)\right)^{-1},
\end{gathered}
$$

where

$$
\begin{gathered}
C_{0}=\widehat{\alpha}_{i, j} F_{i, j}, \quad C_{1}=\widehat{\beta}_{i, j} F_{i, j}+\frac{2 \widehat{\alpha}_{i, j} h_{j} F_{i, j}^{y}}{\pi}, \\
C_{2}=\widehat{\gamma}_{i, j} F_{i, j+1}-\frac{2 \widehat{\delta}_{i, j} h_{j} F_{i, j+1}^{y}}{\pi}, \quad C_{3}=\widehat{\delta}_{i, j} F_{i, j+1}, \\
q_{3}(\varphi)=\widehat{\alpha}_{i, j}(1-\sin \varphi)^{3}+\widehat{\beta}_{i, j} \sin \varphi(1-\sin \varphi)^{2} \\
+\widehat{\gamma}_{i, j} \cos \varphi(1-\cos \varphi)^{2}+\widehat{\delta}_{i, j}(1-\cos \varphi)^{3}, \\
S\left(x_{i+1}, y\right)=\left(D_{0}(1-\sin \varphi)^{3}+D_{1} \sin \varphi(1-\sin \varphi)^{2}\right. \\
\left.+D_{2} \cos \varphi(1-\cos \varphi)^{2}+D_{3}(1-\cos \varphi)^{3}\right) \\
\times\left(q_{4}(\varphi)\right)^{-1},
\end{gathered}
$$

where

$$
\begin{gathered}
D_{0}=\widehat{\alpha}_{i+1, j} F_{i+1, j}, \quad D_{1}=\widehat{\beta}_{i+1, j} F_{i, j+1}+\frac{2 \widehat{\alpha}_{i+1, j} h_{j} F_{i+1, j}^{y}}{\pi}, \\
D_{2}=\widehat{\gamma}_{i+1, j} F_{i+1, j+1}-\frac{2 \widehat{\delta}_{i+1, j} h_{j} F_{i+1, j+1}^{y}}{\pi},
\end{gathered}
$$

$$
\begin{gathered}
D_{3}=\widehat{\delta}_{i+1, j} F_{i+1, j+1}, \\
q_{4}(\varphi)=\widehat{\alpha}_{i+1, j}(1-\sin \varphi)^{3}+\widehat{\beta}_{i+1, j} \sin \varphi(1-\sin \varphi)^{2} \\
+\widehat{\gamma}_{i+1, j} \cos \varphi(1-\cos \varphi)^{2}+\widehat{\delta}_{i+1, j}(1-\cos \varphi)^{3} .
\end{gathered}
$$

\section{Monotone Curve Interpolation}

Monotonicity is a crucial shape property of data and it emanates from many physical phenomenon, engineering problems, scientific applications, and so forth, for instance, dose response curve in biochemistry and pharmacology, approximation of couples and quasi couples in statistics, empirical option pricing model in finance, consumption function in economics, and so forth. Therefore, it is customary that the resulting interpolating curve must retain the monotone shape of data.

In this section, constraints on shape parameters in the description of rational trigonometric cubic function (1) have been developed to preserve $2 \mathrm{D}$ monotone data.

Let $\left\{\left(x_{i}, f_{i}\right), i=0,1,2, \ldots, n\right\}$ be the monotone data defined over the interval $[a, b]$; that is,

$$
\begin{gathered}
f_{i}<f_{i+1}, \quad \Delta_{i}=\frac{f_{i+1}-f_{i}}{h_{i}}>0, \quad i=0,1,2, \ldots, n-1, \\
d_{i}>0, \quad i=0,1,2, \ldots, n .
\end{gathered}
$$

The curve will be monotone if the rational trigonometric cubic function (1) satisfies the condition

$$
S_{i}^{\prime}(x), \quad \forall x \in\left[x_{i}, x_{i+1}\right], i=0,1,2, \ldots, n-1 .
$$

Now, we have

$$
\begin{aligned}
S_{i}^{\prime}(x) & \\
= & \frac{\pi}{2 h_{i}\left(q_{i}(\theta)\right)^{2}} \\
\times & \left\{(1-\sin \theta)^{4} \sin \theta \cos \theta B_{0}\right. \\
& +\cos ^{2} \theta(1-\cos \theta)^{2}(1-\sin \theta)^{2} B_{1} \\
& +\cos \theta(1-\cos \theta)^{3}(1-\sin \theta)^{2} B_{2} \\
& +\cos \theta(1-\sin \theta)^{5} B_{3}+\sin \theta \cos ^{2} \theta(1-\cos \theta)^{2} \\
& \times(1-\sin \theta) B_{4}+\sin \theta \cos \theta(1-\sin \theta)(1-\cos \theta)^{3} B_{5} \\
& +\sin \theta(1-\cos \theta)^{2}(1-\sin \theta)^{3} B_{6} \\
& +\sin { }^{2} \theta(1-\sin \theta)^{2}(1-\cos \theta)^{2} B_{7} \\
& +\sin \theta \cos \theta(1-\cos \theta)^{4} B_{8}+\sin \theta(1-\cos \theta)^{5} B_{9} \\
& +\sin \theta \cos \theta(1-\cos \theta)(1-\sin \theta)^{3} B_{10} \\
& \left.+\sin ^{2} \theta \cos \theta(1-\cos \theta)(1-\sin \theta)^{2} B_{11}\right\},
\end{aligned}
$$


where

$$
\begin{gathered}
B_{0}=\frac{2 h_{i} d_{i} \alpha_{i}^{2}}{\pi}, \\
B_{1}=\left(3 \alpha_{i} \gamma_{i}-\beta_{i} \delta_{i}\right) \Delta_{i}+\frac{2 d_{i} \alpha_{i} \gamma_{i}}{\pi}-\left(3 \alpha_{i}-\beta_{i}\right) \frac{2 \delta_{i} d_{i+1}}{\pi}, \\
B_{2}=\left(\beta_{i}-3 \alpha_{i}\right) \Delta_{i}-\frac{2 d_{i} \alpha_{i}}{\pi}, \quad B_{3}=\frac{2 h_{i} \alpha_{i} d_{i}}{\pi}, \\
B_{4}=2 \beta_{i} \gamma_{i} \Delta_{i}-\frac{4 \delta_{i} \beta_{i} d_{i+1}}{\pi}-\frac{4 \alpha_{i} \gamma_{i} d_{i}}{\pi}, \\
B_{5}=\beta_{i} \Delta_{i}-\frac{2 \alpha_{i} d_{i}}{\pi}, \\
B_{6}=\left(3 \delta_{i}-\gamma_{i}\right) \Delta_{i}+\frac{2 \alpha_{i} d_{i+1}}{\pi}, \\
\beta_{i}\left(\gamma_{i}-3 \delta_{i}\right) \Delta_{i}+\frac{2 \alpha_{i} \gamma_{i} d_{i}}{\pi}+\left(\beta_{i}-3 \alpha_{i}\right) \frac{2 \delta_{i} d_{i+1}}{\pi}, \\
B_{8}=\frac{2 h_{i} \delta_{i} d_{i+1}}{\pi}, \\
B_{9}=\frac{2 h_{i} \delta_{i} d_{i+1}}{\pi}, \\
B_{10}=\gamma_{i} \Delta_{i}-\frac{2 \delta_{i} d_{i+1}}{\pi}, \\
B_{11}=\beta_{i} \gamma_{i} \Delta_{i}-\frac{2 \beta_{i} \delta_{i} d_{i+1}}{\pi}-\frac{2 \alpha_{i} \gamma_{i} d_{i}}{\pi} .
\end{gathered}
$$

The denominator in (16) is a squared quantity, thus, positive. Hence, monotonicity of rational trigonometric cubic spline depends upon the positivity of numerator which can be attained if the coefficients $B_{i}, i=0,1,2, \ldots, 11$ of the trigonometric basis functions are all positive. This yields the following result:

$$
\beta_{i}>\frac{2 \alpha_{i} d_{i}}{\pi \Delta_{i}}, \quad \gamma_{i}>\frac{2 \delta_{i} d_{i+1}}{\pi \Delta_{i}} .
$$

The above discussion can be summarized as follows.

Theorem 1. The $C^{1}$ piecewise trigonometric rational cubic function (1) preserves the monotonicity of monotone data if in each subinterval $I_{i}=\left[x_{i}, x_{i+1}\right]$, the parameters $\beta_{i}$ and $\gamma_{i}$ satisfy the following sufficient conditions:

$$
\beta_{i}>\frac{2 \alpha_{i} d_{i}}{\pi \Delta_{i}}, \quad \gamma_{i}>\frac{2 \delta_{i} d_{i+1}}{\pi \Delta_{i}} .
$$

The above constraints can be rearranged as

$$
\begin{array}{ll}
\beta_{i}=u_{i}+\max \left\{0, \frac{2 \alpha_{i} d_{i}}{\pi \Delta_{i}}\right\}, & u_{i}>0 ; \\
\gamma_{i}=v_{i}+\max \left\{0, \frac{2 \delta_{i} d_{i+1}}{\pi \Delta_{i}}\right\}, & v_{i}>0 .
\end{array}
$$

Algorithm 2.

Step 1. Take a monotone data set $\left\{\left(x_{i}, f_{i}\right): i=0,1,2, \ldots, n\right\}$.

Step 2. Use the Arithmetic Mean Method [11] to estimate the derivatives $d_{i}$ 's at knots $x_{i}$ 's (note: Step 2 is only applicable if data is not provided with derivatives).

Step 3. Compute the values of parameters $\beta_{i}$ 's and $\gamma_{i}$ 's using Theorem 1 .

Step 4. Substitute the values of variables from Steps 13 in rational trigonometric cubic function (1) to visualize monotone curve through monotone data.

\section{Monotone Surface Interpolation}

Let $\left\{\left(x_{i}, y_{j}, F_{i, j}\right), i=0,1,2, \ldots, n-1 ; j=0,1,2, \ldots, m-1\right\}$ be the monotone data set defined over the rectangular mesh $I=\left[x_{i}, x_{i+1}\right] \times\left[y_{j}, y_{j+1}\right]$ such that

$$
\begin{array}{cc}
F_{i, j}<F_{i+1, j}, & F_{i, j}<F_{i, j+1}, \\
F_{i, j}^{x}>0, & F_{i, j}^{y}>0, \\
\Delta_{i, j}>0, & \widehat{\Delta}_{i, j}>0 .
\end{array}
$$

Now, surface patch (4) is monotone if the boundary curves defined in (6)-(12) are monotone.

Now, $S\left(x, y_{j}\right)$ is monotone if $S_{i}^{\prime}\left(x, y_{j}\right)>0$, where

$$
\begin{aligned}
& S_{i}^{\prime}\left(x, y_{j}\right) \\
&=\frac{\pi}{2 h_{i}\left(q_{1}(\theta)\right)^{2}} \\
& \times\left\{(1-\sin \theta)^{4} \sin \theta \cos \theta R_{0}\right. \\
& \quad+\cos ^{2} \theta(1-\cos \theta)^{2}(1-\sin \theta)^{2} R_{1} \\
& \quad+\cos \theta(1-\cos \theta)^{3}(1-\sin \theta)^{2} R_{2} \\
& \quad+\cos \theta(1-\sin \theta)^{5} R_{3} \\
& \quad+\sin \theta \cos { }^{2} \theta(1-\cos \theta)^{2}(1-\sin \theta) R_{4} \\
& \quad+\sin \theta \cos \theta(1-\sin \theta)(1-\cos \theta)^{3} R_{5} \\
& \quad+\sin \theta(1-\cos \theta)^{2}(1-\sin \theta)^{3} R_{6} \\
& \quad+\sin { }^{2} \theta(1-\sin \theta)^{2}(1-\cos \theta)^{2} R_{7} \\
& \quad+\sin \theta \cos \theta(1-\cos \theta)^{4} R_{8}+\sin \theta(1-\cos \theta)^{5} R_{9} \\
& \quad+\sin \theta \cos \theta(1-\cos \theta)(1-\sin \theta)^{3} R_{10} \\
&\left.\quad+\sin { }^{2} \theta \cos \theta(1-\cos \theta)(1-\sin \theta)^{2} R_{11}\right\},
\end{aligned}
$$


with

$$
\begin{gathered}
R_{0}=\frac{2 h_{i} F_{i, j}^{x} \alpha_{i, j}^{2}}{\pi}, \\
R_{1}=\left(3 \alpha_{i, j} \gamma_{i, j}-\beta_{i, j} \delta_{i, j}\right) \Delta_{i, j}+\frac{2 F_{i, j}^{x} \alpha_{i, j} \gamma_{i, j}}{\pi} \\
-\left(3 \alpha_{i, j}-\beta_{i, j}\right) \frac{2 \delta_{i} F_{i+1, j}^{x}}{\pi}, \\
R_{2}=\left(\beta_{i, j}-3 \alpha_{i, j}\right) \Delta_{i, j}-\frac{2 F_{i, j}^{x} \alpha_{i, j}}{\pi}, \\
R_{4}=2 \beta_{i, j} \gamma_{i, j} \Delta_{i, j}-\frac{4 \delta_{i, j} \beta_{i, j} F_{i+1, j}^{x}}{\pi}-\frac{4 \alpha_{i, j} \gamma_{i, j} F_{i, j}^{x}}{\pi}, \\
R_{5}=\beta_{i, j} \Delta_{i, j}-\frac{2 h_{i} \alpha_{i, j} F_{i, j}^{x}}{\pi}, \\
R_{11}=\beta_{i, j} \gamma_{i, j} \Delta_{i, j}-\frac{2 \beta_{i, j} \delta_{i, j} F_{i+1, j}^{x}}{\pi}-\frac{2 \alpha_{i, j} \gamma_{i, j} F_{i, j}^{x}}{\pi} . \\
R_{7}=\beta_{i, j}\left(\gamma_{i, j}-\gamma_{i, j}\right) \Delta_{i, j}+\frac{2 \alpha_{i, j} F_{i+1, j}^{x}}{\pi}, \\
R_{8}=\frac{\left.2 \delta_{i, j}\right) \Delta_{i, j}+\frac{2 \alpha_{i, j} \gamma_{i, j} F_{i, j}^{x}}{\pi}}{\pi} \\
+\left(\beta_{i, j}-3 \alpha_{i, j}\right) \frac{2 \delta_{i, j} F_{i+1, j}^{x}}{\pi}, \\
R_{i+1, j}^{x}, \\
R_{9}=\frac{2 h_{i} \delta_{i, j} F_{i+1, j}^{x}}{\pi}, \\
R_{i, j}-\frac{2 \delta_{i, j} F_{i+1, j}^{x}}{\pi},
\end{gathered}
$$

Now the positivity of $S_{i}^{\prime}\left(x, y_{j}\right)$ entirely depends on $R_{i}, i=$ $0,1,2 \ldots, 11$. The denominator in (22) is always positive. Since the parameter $\theta$ lies in first quadrant therefore the trigonometric basis functions will be positive also. This yields the following constraints on the free parameters:

$$
\beta_{i, j}>\frac{2 \alpha_{i, j} F_{i, j}^{x}}{\pi \Delta_{i, j}}, \quad \gamma_{i, j}>\frac{2 \delta_{i, j} F_{i+1, j}^{x}}{\pi \Delta_{i, j}} .
$$

$S\left(x, y_{j+1}\right)$ is monotone if

$$
S_{i}^{\prime}\left(x, y_{j+1}\right)>0
$$

where

$$
\begin{aligned}
& S_{i}^{\prime}\left(x, y_{j+1}\right) \\
& =\frac{\pi}{2 h_{i}\left(q_{2}(\theta)\right)^{2}} \\
& \quad \times\left\{(1-\sin \theta)^{4} \sin \theta \cos \theta T_{0}\right. \\
& \quad+\cos ^{2} \theta(1-\cos \theta)^{2}(1-\sin \theta)^{2} T_{1} \\
& \quad+\cos \theta(1-\cos \theta)^{3}(1-\sin \theta)^{2} T_{2} \\
& \quad+\cos \theta(1-\sin \theta)^{5} T_{3} \\
& \quad+\sin \theta \cos { }^{2} \theta(1-\cos \theta)^{2}(1-\sin \theta) T_{4} \\
& \quad+\sin \theta \cos \theta(1-\sin \theta)(1 \cos \theta)^{3} T_{5} \\
& \quad+\sin \theta(1-\cos \theta)^{2}(1-\sin \theta)^{3} T_{6} \\
& \quad+\sin { }^{2} \theta(1-\sin \theta)^{2}(1-\cos \theta)^{2} T_{7} \\
& \quad+\sin \theta \cos \theta(1-\cos \theta)^{4} T_{8}+\sin \theta(1 \cos \theta)^{5} T_{9} \\
& \quad+\sin \theta \cos \theta(1-\cos \theta)(1-\sin \theta)^{3} T_{10} \\
& \left.\quad+\sin { }^{2} \theta \cos \theta(1-\cos \theta)(1-\sin \theta)^{2} T_{11}\right\},
\end{aligned}
$$

with

$$
\begin{gathered}
T_{0}=\frac{2 h_{i} F_{i, j+1}^{x} \alpha_{i, j+1}^{2}}{\pi}, \\
T_{1}=\left(3 \alpha_{i, j+1} \gamma_{i, j+1}-\beta_{i, j+1} \delta_{i, j+1}\right) \Delta_{i, j+1} \\
+\frac{2 F_{i, j+1}^{x} \alpha_{i, j+1} \gamma_{i, j+1}}{\pi} \\
-\left(3 \alpha_{i, j+1}-\beta_{i, j+1}\right) \frac{2 \delta_{i, j+1} F_{i+1, j+1}^{x}}{\pi}, \\
T_{2}=\left(\beta_{i, j+1}-3 \alpha_{i, j+1}\right) \Delta_{i, j+1}-\frac{2 F_{i, j+1}^{x} \alpha_{i, j+1}}{\pi}, \\
T_{4}=2 \beta_{i, j+1} \gamma_{i, j+1} \Delta_{i, j+1} \\
-\frac{4 \delta_{i, j+1} \beta_{i, j+1} F_{i+1, j+1}^{x}-\frac{4 \alpha_{i, j+1} \gamma_{i, j+1} F_{i, j+1}^{x}}{\pi}}{\pi} \\
T_{6}=\left(3 \delta_{i, j+1}-\gamma_{i, j+1}\right) \Delta_{i, j+1}+\frac{2 \alpha_{i, j+1} F_{i+1, j+1}^{x}}{\pi}, \\
T_{5}=\beta_{i, j+1} \Delta_{i, j+1}-\frac{2 \alpha_{i, j+1} F_{i, j+1}^{x}}{\pi}, \\
\pi
\end{gathered}
$$




$$
\begin{gathered}
T_{7}=\beta_{i, j+1}\left(\gamma_{i, j+1}-3 \delta_{i, j+1}\right) \Delta_{i, j+1}+\frac{2 \alpha_{i, j+1} \gamma_{i, j+1} F_{i, j+1}^{x}}{\pi} \\
+\left(\beta_{i, j+1}-3 \alpha_{i, j+1}\right) \frac{2 \delta_{i, j+1} F_{i+1, j+1}^{x}}{\pi}, \\
T_{8}=\frac{2 h_{i} \delta_{i, j+1} F_{i, j+1}^{x}}{\pi}, \quad T_{9}=\frac{2 h_{i} \delta_{i, j+1} F_{i+1, j+1}^{x}}{\pi}, \\
T_{11}=\beta_{i, j+1} \gamma_{i, j+1} \Delta_{i, j+1}-\frac{2 \beta_{i, j+1} \delta_{i, j+1} F_{i+1, j+1}^{x}}{\pi} \\
-\frac{2 \alpha_{i, j+1} \gamma_{i, j+1} F_{i, j+1}^{x}}{\pi} .
\end{gathered}
$$

The denominator in (26) is always positive. Moreover, the trigonometric basis functions are also positive for $0 \leq$ $\theta \leq \pi / 2$. It follows that the positivity of $S_{i}^{\prime}\left(x, y_{j+1}\right)$ entirely depends upon $T_{i}, i=0,1,2 \ldots, 11$. This yields the following constraints on the free parameters:

$$
\beta_{i, j+1}>\frac{2 \alpha_{i, j+1} F_{i, j+1}^{x}}{\pi \Delta_{i, j+1}}, \quad \gamma_{i, j+1}>\frac{2 \delta_{i, j+1} F_{i+1, j+1}^{x}}{\pi \Delta_{i, j+1}} .
$$

$S\left(x_{i}, y\right)$ is monotone if $S_{i}^{\prime}\left(x_{i}, y\right)>0$. We have

$$
\begin{aligned}
S_{i}^{\prime}\left(x_{i}, y\right) & \frac{\pi}{2 h_{i}\left(q_{3}(\varphi)\right)^{2}} \\
\times & \left\{(1-\sin \varphi)^{4} \sin \varphi \cos \varphi U_{0}\right. \\
& +\cos ^{2} \varphi(1-\cos \varphi)^{2}(1-\sin \varphi)^{2} U_{1} \\
& +\cos \varphi(1-\cos \varphi)^{3}(1-\sin \varphi)^{2} U_{2} \\
& +\cos \varphi(1-\sin \varphi)^{5} U_{3} \\
& +\sin \varphi \cos { }^{2} \varphi(1-\cos \varphi)^{2}(1-\sin \varphi) U_{4} \\
& +\sin ^{2} \cos \varphi(1-\sin \varphi)(1 \cos \varphi)^{3} U_{5} \\
& +\sin (1-\cos \varphi)^{2}(1-\sin \varphi)^{3} U_{6} \\
& +\sin ^{2} \varphi(1-\sin \varphi)^{2}(1-\cos \varphi)^{2} U_{7} \\
& +\sin ^{2} \cos \varphi(1-\cos \varphi)^{4} U_{8}+\sin \varphi(1 \cos \varphi)^{5} U_{9} \\
& +\sin \varphi \cos \varphi(1-\cos \varphi)(1-\sin \varphi)^{3} U_{10} \\
& \left.+\sin ^{2} \varphi \cos \varphi(1-\cos \varphi)(1-\sin \varphi)^{2} U_{11}\right\}
\end{aligned}
$$

where

$$
\begin{aligned}
& U_{0}=\frac{2 h_{j} F_{i, j}^{y} \widehat{\alpha}_{i, j}^{2}}{\pi}, \\
& U_{1}=\left(3 \widehat{\alpha}_{i, j} \widehat{\gamma}_{i, j}-\widehat{\beta}_{i, j} \widehat{\delta}_{i, j}\right) \widehat{\Delta}_{i, j}+\frac{2 F_{i, j}^{y} \widehat{\alpha}_{i, j} \widehat{\gamma}_{i, j}}{\pi} \\
& -\left(3 \widehat{\alpha}_{i, j}-\widehat{\beta}_{i, j}\right) \frac{2 \widehat{\delta}_{i, j} F_{i, j+1}^{y}}{\pi} \\
& U_{2}=\left(\widehat{\beta}_{i, j}-3 \widehat{\alpha}_{i, j}\right) \widehat{\Delta}_{i, j}-\frac{2 F_{i, j}^{y} \widehat{\alpha}_{i, j}}{\pi}, \\
& U_{3}=\frac{2 h_{j} \widehat{\alpha}_{i, j} F_{i, j}^{y}}{\pi}, \\
& U_{4}=2 \widehat{\beta}_{i, j} \widehat{\gamma}_{i, j} \widehat{\Delta}_{i, j}-\frac{4 \widehat{\delta}_{i, j} \widehat{\beta}_{i, j} F_{i, j+1}^{y}}{\pi}-\frac{4 \widehat{\alpha}_{i, j} \widehat{\gamma}_{i, j} F_{i, j}^{y}}{\pi}, \\
& U_{5}=\widehat{\beta}_{i, j} \widehat{\Delta}_{i, j}-\frac{2 \widehat{\alpha}_{i, j} F_{i, j}^{y}}{\pi}, \\
& U_{6}=\left(3 \widehat{\delta}_{i, j}-\widehat{\gamma}_{i, j}\right) \widehat{\Delta}_{i, j}+\frac{2 \widehat{\alpha}_{i, j} F_{i, j+1}^{y}}{\pi}, \\
& U_{7}=\widehat{\beta}_{i, j}\left(\widehat{\gamma}_{i, j}-3 \widehat{\delta}_{i, j}\right) \widehat{\Delta}_{i, j}+\frac{2 \widehat{\alpha}_{i, j} \widehat{\gamma}_{i, j} F_{i, j}^{y}}{\pi} \\
& +\left(\widehat{\beta}_{i, j}-3 \widehat{\alpha}_{i, j}\right) \frac{2 \widehat{\delta}_{i, j} F_{i, j+1}^{y}}{\pi}, \\
& U_{8}=\frac{2 h_{j} \widehat{\delta}_{i, j} F_{i, j+1}^{y}}{\pi} \\
& U_{9}=\frac{2 h_{j} \widehat{\delta}_{i, j} F_{i, j+1}^{y}}{\pi}, \quad U_{10}=\widehat{\gamma}_{i, j} \widehat{\Delta}_{i, j}-\frac{2 \widehat{\delta}_{i, j} F_{i, j+1}^{y}}{\pi} \\
& U_{11}=\widehat{\beta}_{i, j} \widehat{\gamma}_{i, j} \widehat{\Delta}_{i, j}-\frac{2 \widehat{\beta}_{i, j} \widehat{\delta}_{i, j} F_{i, j+1}^{y}}{\pi}-\frac{2 \widehat{\alpha}_{i, j} \widehat{\gamma}_{i, j} F_{i, j}^{y}}{\pi} .
\end{aligned}
$$

Since the denomoinator of (29) is always positive and trigonometric basis functions are positive for so the positivity of $0 \leq \varphi \leq \pi / 2$. It follows that the positivity of $S_{i}^{\prime}\left(x_{i+1}, y\right)$ entirely depends upon $U_{i}, i=0,1,2 \ldots, 11$. This yields the following constraints on the free parameters:

$$
\widehat{\beta}_{i, j}>\frac{2 \widehat{\alpha}_{i, j} F_{i, j}^{y}}{\pi \widehat{\Delta}_{i, j}}, \quad \widehat{\gamma}_{i, j}>\frac{2 \widehat{\delta}_{i, j} F_{i, j+1}^{y}}{\pi \widehat{\Delta}_{i, j}} .
$$


TABLE 1: The varying ability of hemoglobin to carry oxygen.

\begin{tabular}{lccccc}
\hline $\begin{array}{l}\text { Partial pressure of oxygen } \\
(\mathrm{kPa})\end{array}$ & 0 & 2 & 8 & 10 & 18 \\
\hline $\begin{array}{l}\text { Saturation of hemoglobin } \\
(\%)\end{array}$ & 0 & 70 & 91 & 91 & 110 \\
\hline
\end{tabular}

TABLE 2: The varying ability of myoglobin.

\begin{tabular}{lccccc}
\hline $\begin{array}{l}\text { Partial pressure of oxygen } \\
(\mathrm{kPa})\end{array}$ & 0 & 4 & 6 & 8 & 10 \\
\hline $\begin{array}{l}\text { Saturation of myoglobin } \\
(\%)\end{array}$ & 0 & 100 & 100 & 100 & 115 \\
\hline
\end{tabular}

TABLE 3: Numerical results corresponding to Figure 2.

\begin{tabular}{cccccc}
\hline$i$ & 1 & 2 & 3 & 4 & 5 \\
\hline$d_{i}$ & 50.9065 & 19.6819 & 0 & 0 & 5.7983 \\
$\beta_{i}$ & 35.01 & 7.17 & 0 & 0.01 & - \\
$\gamma_{i}$ & 0.1890 & 0.0100 & 0 & 0.7871 & - \\
\hline
\end{tabular}

TABLE 4: Numerical results corresponding to Figure 4.

\begin{tabular}{cccccc}
\hline$i$ & 1 & 2 & 3 & 4 & 5 \\
\hline$d_{i}$ & 56.25 & 0 & 0 & 0 & 15 \\
$\beta_{i}$ & 2.8748 & 9.3179 & 0 & 0.01 & - \\
$\gamma_{i}$ & 0.01 & 0.01 & 0 & 0.6466 & - \\
\hline
\end{tabular}

$S\left(x_{i+1}, y\right)$ is monotone if $S_{i}^{\prime}\left(x_{i+1}, y\right)>0$. We have

$$
\begin{aligned}
S_{i}^{\prime}\left(x_{i+1}, y\right) & \frac{\pi}{2 h_{i}\left(q_{4}(\varphi)\right)^{2}} \\
\quad \times\{ & (1-\sin \varphi)^{4} \sin \varphi \cos \varphi V_{0} \\
& +\cos ^{2} \varphi(1-\cos \varphi)^{2}(1-\sin \varphi)^{2} V_{1} \\
& +\cos \varphi(1-\cos \varphi)^{3}(1-\sin \varphi)^{2} V_{2} \\
& +\cos \varphi(1-\sin \varphi)^{5} V_{3} \\
& +\sin \varphi \cos { }^{2} \varphi(1-\cos \varphi)^{2}(1-\sin \varphi) V_{4} \\
& +\sin \varphi \cos \varphi(1-\sin \varphi)(1 \cos \varphi)^{3} V_{5} \\
& +\sin \varphi(1-\cos \varphi)^{2}(1-\sin \varphi)^{3} V_{6} \\
& +\sin ^{2} \varphi(1-\sin \varphi)^{2}(1-\cos \varphi)^{2} V_{7} \\
& +\sin \varphi \cos \varphi(1-\cos \varphi)^{4} V_{8}+\sin \varphi(1 \cos \varphi)^{5} V_{9} \\
& +\sin \varphi \cos \varphi(1-\cos \varphi)(1-\sin \varphi)^{3} V_{10} \\
& \left.+\sin ^{2} \varphi \cos \varphi(1-\cos \varphi)(1-\sin \varphi)^{2} V_{11}\right\}
\end{aligned}
$$

where

$$
\begin{aligned}
& V_{0}=\frac{2 h_{j} F_{i+1, j}^{y} \widehat{\alpha}_{i+1, j}^{2}}{\pi}, \\
& V_{1}=\left(3 \widehat{\alpha}_{i+1, j} \widehat{\gamma}_{i, j}-\widehat{\beta}_{i+1, j} \widehat{\delta}_{i+1, j}\right) \widehat{\Delta}_{i+1, j}+\frac{2 F_{i+1, j}^{y} \widehat{\alpha}_{i+1, j} \widehat{\gamma}_{i+1, j}}{\pi} \\
& -\left(3 \widehat{\alpha}_{i+1, j}-\widehat{\beta}_{i+1, j}\right) \frac{2 \widehat{\delta}_{i+1, j} F_{i+1, j+1}^{y}}{\pi}, \\
& V_{3}=\frac{2 h_{j} \widehat{\alpha}_{i+1, j} F_{i+1, j}^{y}}{\pi}, \\
& V_{4}=2 \widehat{\beta}_{i+1, j} \widehat{\gamma}_{i+1, j} \widehat{\Delta}_{i+1, j} \\
& -\frac{4 \widehat{\delta}_{i+1, j} \widehat{\beta}_{i+1, j} F_{i+1, j+1}^{y}}{\pi}-\frac{4 \widehat{\alpha}_{i+1, j} \widehat{\gamma}_{i+1, j} F_{i+1, j}^{y}}{\pi}, \\
& V_{5}=\widehat{\beta}_{i+1, j} \widehat{\Delta}_{i+1, j}-\frac{2 \widehat{\alpha}_{i+1, j} F_{i+1, j}^{x}}{\pi}, \\
& V_{6}=\left(3 \widehat{\delta}_{i+1, j}-\widehat{\gamma}_{i+1, j}\right) \widehat{\Delta}_{i, j}+\frac{2 \widehat{\alpha}_{i+1, j} F_{i+1, j+1}^{x}}{\pi}, \\
& V_{7}=\widehat{\beta}_{i+1, j}\left(\widehat{\gamma}_{i+1, j}-3 \widehat{\delta}_{i+1, j}\right) \widehat{\Delta}_{i+1, j}+\frac{2 \widehat{\alpha}_{i+1, j} \widehat{\gamma}_{i+1, j} F_{i, j}^{y}}{\pi} \\
& +\left(\widehat{\beta}_{i+1, j}-3 \widehat{\alpha}_{i+1, j}\right) \frac{2 \widehat{\delta}_{i, j} F_{i+1, j+1}^{y}}{\pi} \\
& V_{8}=\frac{2 h_{j} \widehat{\delta}_{i+1, j} F_{i+1, j+1}^{y}}{\pi}, \quad V_{9}=\frac{2 h_{j} \widehat{\delta}_{i+1, j} F_{i+1, j+1}^{x}}{\pi}, \\
& V_{10}=\widehat{\gamma}_{i+1, j} \widehat{\Delta}_{i+1, j}-\frac{2 \widehat{\delta}_{i+1, j} F_{i+1, j+1}^{y}}{\pi}, \\
& V_{11}=\widehat{\beta}_{i+1, j} \widehat{\gamma}_{i+1, j} \widehat{\Delta}_{i+1, j}-\frac{2 \widehat{\beta}_{i+1, j} \widehat{\delta}_{i+1, j} F_{i+1, j+1}^{y}}{\pi} \\
& -\frac{2 \widehat{\alpha}_{i+1, j} \widehat{\gamma}_{i+1, j} F_{i+1, j}^{x}}{\pi} .
\end{aligned}
$$

Finally, $S_{i}^{\prime}\left(x_{i+1}, y\right)$ is positive if $V_{i}, i=0,1,2 \ldots, 11$ are positive. This yields the following constraints on the free parameters:

$$
\widehat{\beta}_{i+1, j}>\frac{2 \widehat{\alpha}_{i+1, j} F_{i+1, j}^{y}}{\pi \widehat{\Delta}_{i+1, j}}, \quad \widehat{\gamma}_{i+1, j}>\frac{2 \widehat{\delta}_{i+1, j} F_{i+1, j+1}^{y}}{\pi \widehat{\Delta}_{i+1, j}} .
$$

The above discussion can be put forward as the following theorem.

Theorem 3. The bicubic partially blended rational trigonometric function defined in (4) visualizes monotone data in view of the monotone surface if in each rectangular grid $I=\left[x_{i}, x_{i+1}\right] \times\left[y_{j}, y_{j+1}\right]$, free parameters $\beta_{i, j}, \gamma_{i, j}$, 
TABLe 5: A 3D monotone data set.

\begin{tabular}{lccccrr}
\hline$y / x$ & 1 & 2 & 3 & 4 & 5 \\
\hline 1 & 0.3202 & 0.5385 & 0.7762 & 1.0198 & 1.2659 & 1.5133 \\
2 & 0.4717 & 0.6403 & 0.8500 & 1.0770 & 1.3124 & 1.5524 \\
3 & 0.6500 & 0.7810 & 0.9605 & 1.2862 & 1.3865 & 1.6155 \\
4 & 0.8382 & 0.9434 & 1.0966 & 1.4142 & 1.4841 & 1.6008 \\
5 & 1.0308 & 1.1180 & 1.2500 & 1.5620 & 1.7328 \\
6 & 1.2258 & 1.3000 & 1.4151 & & 1.90209 \\
\hline
\end{tabular}

TABLe 6: A 3D monotone data set.

\begin{tabular}{lccccrr}
\hline$y / x$ & 1 & 2 & 3 & 4 & 5 & 3 \\
\hline 1 & 0.6931 & 1.6094 & 2.3026 & 2.8332 & 3.2581 & 3.6109 \\
2 & 1.6094 & 2.0794 & 2.5649 & 2.9957 & 3.3673 & 3.6889 \\
3 & 2.3026 & 2.5649 & 3.2189 & 3.4657 & 3.8067 \\
4 & 2.8332 & 2.9957 & 3.5264 & 3.7136 & 3.9136 & 3.9512 \\
5 & 3.2581 & 3.3673 & 3.8067 & 3.9512 & 4.1109 \\
6 & 3.6109 & 3.6889 & & & 4.2767 \\
\hline
\end{tabular}

$\beta_{i, j+1}, \gamma_{i, j+1}, \widehat{\beta}_{i, j}, \widehat{\gamma}_{i, j}, \widehat{\beta}_{i+1, j}, \widehat{\gamma}_{i+1, j}$ satisfy the following constraints:

$$
\begin{aligned}
\beta_{i, j}>\frac{2 \alpha_{i, j} F_{i, j}^{x}}{\pi \Delta_{i, j}}, & \gamma_{i, j}>\frac{2 \delta_{i, j} F_{i+1, j}^{x}}{\pi \Delta_{i, j}}, \\
\beta_{i, j+1}>\frac{2 \alpha_{i, j+1} F_{i, j+1}^{x}}{\pi \Delta_{i, j+1}}, & \gamma_{i, j+1}>\frac{2 \delta_{i, j+1} F_{i+1, j+1}^{x}}{\pi \Delta_{i, j+1}}, \\
\widehat{\beta}_{i, j}>\frac{2 \widehat{\alpha}_{i, j} F_{i, j}^{y}}{\pi \widehat{\Delta}_{i, j}}, & \widehat{\gamma}_{i, j}>\frac{2 \widehat{\delta}_{i, j} F_{i, j+1}^{y}}{\pi \widehat{\Delta}_{i, j}}, \\
\widehat{\beta}_{i+1, j}>\frac{2 \widehat{\alpha}_{i+1, j} F_{i+1, j}^{y}}{\pi \widehat{\Delta}_{i+1, j}}, & \widehat{\gamma}_{i+1, j}>\frac{2 \widehat{\delta}_{i+1, j} F_{i+1, j+1}^{y}}{\pi \widehat{\Delta}_{i+1, j}} .
\end{aligned}
$$

The above constraints are rearranged as

$$
\begin{gathered}
\beta_{i, j}=l_{i, j}+\max \left\{0, \frac{2 \alpha_{i, j} F_{i, j}^{x}}{\pi \Delta_{i, j}}\right\}, \quad l_{i, j}>0, \\
\gamma_{i, j}=m_{i, j}+\max \left\{\frac{2 \delta_{i, j} F_{i+1, j}^{x}}{\pi \Delta_{i, j}}\right\}, \quad m_{i, j}>0, \\
\beta_{i, j+1}=n_{i, j}+\max \left\{\frac{2 \alpha_{i, j+1} F_{i, j+1}^{x}}{\pi \Delta_{i, j+1}}\right\}, \quad n_{i, j}>0, \\
\gamma_{i, j+1}=o_{i, j}+\max \left\{\frac{2 \delta_{i, j+1} F_{i+1, j+1}^{x}}{\pi \Delta_{i, j+1}}\right\}, \quad o_{i, j}>0, \\
\widehat{\beta}_{i, j}=r_{i, j}+\max \left\{\frac{2 \widehat{\alpha}_{i, j} F_{i, j}^{y}}{\pi \widehat{\Delta}_{i, j}}\right\}, \quad r_{i, j}>0,
\end{gathered}
$$

$$
\begin{gathered}
\widehat{\gamma}_{i, j}=s_{i, j}+\max \left\{\frac{2 \widehat{\delta}_{i, j} F_{i, j+1}^{y}}{\pi \widehat{\Delta}_{i, j}}\right\}, \quad s_{i, j}>0, \\
\widehat{\beta}_{i+1, j}=t_{i, j}+\max \left\{\frac{2 \widehat{\alpha}_{i+1, j} F_{i+1, j}^{y}}{\pi \widehat{\Delta}_{i+1, j}}\right\}, \quad t_{i, j}>0, \\
\widehat{\gamma}_{i+1, j}=u_{i, j}+\max \left\{\frac{2 \widehat{\delta}_{i+1, j} F_{i+1, j+1}^{y}}{\pi \widehat{\Delta}_{i+1, j}}\right\}, \quad u_{i, j}>0 .
\end{gathered}
$$

Algorithm 4.

Step 1. Take a $3 \mathrm{D}$ monotone data set $\left\{\left(x_{i}, y_{j}, F_{i, j}\right), i=\right.$ $0,1,2, \ldots, n ; j=0,1,2, \ldots, m\}$.

Step 2. Use the Arithmetic Mean Method to estimate the derivatives $F_{i, j}^{x}, F_{i, j}^{y}, F_{i, j}^{x y}$ at knots (note: Step 2 is only applicable if data is not provided with derivatives).

Step 3. Compute the values of parameters $\beta_{i, j}, \gamma_{i, j}, \beta_{i, j+1}$, $\gamma_{i, j+1}, \widehat{\beta}_{i, j}, \widehat{\gamma}_{i, j}, \widehat{\beta}_{i+1, j}, \widehat{\gamma}_{i+1, j}$ using Theorem 3 .

Step 4. Substitute the values of variables from Steps 1-3 in rational trigonometric cubic function (4) to visualize monotone surface through monotone data.

\section{Numerical Example}

This section illustrates the monotonicity preserving schemes developed in Sections 4 and 5 with the help of examples. The data in Table 1 is observed by exposing identical samples of hemoglobin to different partial pressures of oxygen which results in varying degree of saturation of hemoglobin with 
TABLe 7: Numerical values corresponding to Figure 6.

\begin{tabular}{|c|c|c|c|c|c|c|}
\hline$\left(x_{i}, y_{j}\right)$ & 1 & 2 & 3 & 4 & 5 & 6 \\
\hline \multicolumn{7}{|c|}{ Numerical values of $F_{i, j}^{x}$} \\
\hline 1 & 0.1382 & 0.0823 & 0.0555 & 0.0413 & 0.0328 & 0.0271 \\
\hline 2 & 0.1649 & 0.1213 & 0.0921 & 0.0732 & 0.0603 & 0.0511 \\
\hline 3 & 0.1832 & 0.1515 & 0.1233 & 0.1018 & 0.0858 & 0.0738 \\
\hline 4 & 0.1904 & 0.1685 & 0.1448 & 0.1240 & 0.1071 & 0.0936 \\
\hline 5 & 0.1938 & 0.1783 & 0.1593 & 0.1407 & 0.1243 & 0.1105 \\
\hline 6 & 0.1962 & 0.1856 & 0.1709 & 0.1550 & 0.1396 & 0.1259 \\
\hline \multicolumn{7}{|c|}{ Numerical values of $F_{i, j}^{y}$} \\
\hline 1 & 0.2087 & 0.2280 & 0.2406 & 0.2448 & 0.2467 & 0.2480 \\
\hline 2 & 0.1481 & 0.1892 & 0.2184 & 0.2312 & 0.2377 & 0.2423 \\
\hline 3 & 0.1068 & 0.1552 & 0.1926 & 0.2130 & 0.2247 & 0.2333 \\
\hline 4 & 0.0813 & 0.1292 & 0.1686 & 0.1937 & 0.2097 & 0.2221 \\
\hline 5 & 0.0649 & 0.1096 & 0.1481 & 0.1754 & 0.1943 & 0.2097 \\
\hline 6 & 0.0538 & 0.0947 & 0.1310 & 0.1588 & 0.1794 & 0.1969 \\
\hline \multicolumn{7}{|c|}{ Numerical values of $\beta_{i, j}$} \\
\hline 1 & 10.9406 & 9.7062 & 9.0177 & 8.6526 & 8.4470 & - \\
\hline 2 & 11.0996 & 10.3406 & 10.0079 & 9.8513 & 9.7684 & - \\
\hline 3 & 11.6858 & 11.1996 & 10.8694 & 10.6747 & 10.5583 & - \\
\hline 4 & 11.8607 & 11.5787 & 11.3235 & 11.1397 & 11.0149 & - \\
\hline 5 & 11.9272 & 11.7583 & 11.5754 & 11.4218 & 11.3048 & - \\
\hline 6 & - & - & - & - & - & - \\
\hline \multicolumn{7}{|c|}{ Numerical values of $\gamma_{i, j}$} \\
\hline 1 & 13.0594 & 14.2938 & 14.9823 & 15.3474 & 15.5530 & - \\
\hline 2 & 12.3315 & 12.9236 & 13.3931 & 13.7011 & 13.8977 & - \\
\hline 3 & 12.1426 & 12.4531 & 12.7625 & 13.0043 & 13.1785 & - \\
\hline 4 & 12.0737 & 12.2518 & 12.4569 & 12.6399 & 12.7863 & - \\
\hline 5 & 12.0728 & 12.2417 & 12.4246 & 12.5782 & 12.6952 & - \\
\hline 6 & - & - & - & - & - & - \\
\hline \multicolumn{7}{|c|}{ Numerical values of $\widehat{\beta}_{i, j}$} \\
\hline 1 & 11.4688 & 11.5120 & 11.8546 & 11.9391 & 11.9689 & - \\
\hline 2 & 10.5384 & 10.8247 & 11.5416 & 11.7866 & 11.8858 & - \\
\hline 3 & 9.7828 & 10.3810 & 11.2336 & 11.6016 & 11.7732 & - \\
\hline 4 & 9.2668 & 10.1222 & 10.9942 & 11.4274 & 11.6537 & - \\
\hline 5 & 8.9258 & 9.9673 & 10.8217 & 11.2811 & 11.5418 & - \\
\hline 6 & - & - & - & - & - & - \\
\hline \multicolumn{7}{|c|}{ Numerical values of $\widehat{\gamma}_{i, j}$} \\
\hline 1 & 12.5312 & 12.1490 & 12.0616 & 12.0312 & 12.0311 & - \\
\hline 2 & 13.4616 & 12.4963 & 12.2213 & 12.1165 & 12.1142 & - \\
\hline 3 & 14.2172 & 12.8787 & 12.4267 & 12.2357 & 12.2268 & - \\
\hline 4 & 14.7332 & 13.2084 & 12.6331 & 12.3675 & 12.3463 & - \\
\hline 5 & 15.0742 & 13.4662 & 12.8168 & 12.4961 & 12.4582 & - \\
\hline 6 & - & - & - & - & - & - \\
\hline \multicolumn{7}{|c|}{ Numerical values of $\beta_{i, j+1}$} \\
\hline 1 & 11.3239 & 10.5207 & 10.0947 & 9.8548 & 9.7100 & - \\
\hline 2 & 12.0640 & 11.6759 & 11.4932 & 11.3965 & 11.3401 & - \\
\hline 3 & 13.0662 & 12.6810 & 12.4538 & 12.3180 & 12.2329 & - \\
\hline 4 & 13.5085 & 13.2108 & 12.9963 & 12.8508 & 12.7519 & - \\
\hline 5 & 13.7180 & 13.5047 & 13.3254 & 13.1890 & 13.0885 & - \\
\hline 6 & - & - & - & - & - & - \\
\hline
\end{tabular}


TABLe 7: Continued.

\begin{tabular}{|c|c|c|c|c|c|c|}
\hline$\left(x_{i}, y_{j}\right)$ & 1 & 2 & 3 & 4 & 5 & 6 \\
\hline \multicolumn{7}{|c|}{ Numerical values of $\gamma_{i, j+1}$} \\
\hline 1 & 15.4850 & 16.2308 & 16.6264 & 16.8491 & 16.9836 & - \\
\hline 2 & 14.0006 & 14.5092 & 14.8428 & 15.0559 & 15.1949 & - \\
\hline 3 & 13.4909 & 13.8260 & 14.0880 & 14.2768 & 14.4104 & - \\
\hline 4 & 13.2728 & 13.4950 & 13.6932 & 13.8518 & 13.9731 & - \\
\hline 5 & 13.2618 & 13.4600 & 13.6264 & 13.7531 & 13.8464 & - \\
\hline 6 & - & - & - & - & - & - \\
\hline \multicolumn{7}{|c|}{ Numerical values of $\widehat{\beta}_{i+1, j}$} \\
\hline 1 & 9.4938 & 11.1409 & 12.5497 & 13.1538 & 13.4520 & - \\
\hline 2 & 8.8689 & 10.3644 & 11.8756 & 12.6696 & 13.1076 & - \\
\hline 3 & 8.6842 & 10.0813 & 11.4747 & 12.3097 & 12.8189 & - \\
\hline 4 & 8.6336 & 10.0176 & 11.2653 & 12.0687 & 12.5971 & - \\
\hline 5 & 8.6325 & 10.0429 & 11.1705 & 11.9192 & 12.4370 & - \\
\hline 6 & - & - & - & - & - & - \\
\hline \multicolumn{7}{|c|}{ Numerical values of $\widehat{\gamma}_{i+1, j}$} \\
\hline 1 & 14.5834 & 13.5377 & 13.2398 & 13.1262 & 13.1238 & - \\
\hline 2 & 15.4020 & 13.9519 & 13.4623 & 13.2553 & 13.2457 & - \\
\hline 3 & 15.9609 & 14.3091 & 13.6858 & 13.3981 & 13.3751 & - \\
\hline 4 & 16.3304 & 14.5884 & 13.8848 & 13.5375 & 13.4964 & - \\
\hline 5 & 16.5779 & 14.7990 & 14.0514 & 13.6641 & 13.6025 & - \\
\hline 6 & - & - & - & - & - & - \\
\hline
\end{tabular}

oxygen. The sample obtaining the highest amount is said to be saturated. The amount of oxygen combined with the remaining samples is taken as percentage of this maximum value. At a low partial pressure of oxygen, the percentage saturation of hemoglobin is very low; that is, hemoglobin is combined with only a very little oxygen. At high partial pressure of oxygen, the percentage saturation of hemoglobin is very high; that is, hemoglobin is combined with large amounts of oxygen, that is, a monotone relation, so the resulting curve must exhibit the same behavior. Figure 1 represents the curve created by assigning random values to free parameters in description of $C^{1}$ rational trigonometric cubic function (1) which does not retain the monotone nature of the data. This impediment is removed by applying monotonicity preserving schemes developed in Section 4 and is shown in Figure 2. It is evident from the figure that this curve preserves the monotone shape of hemoglobin dissociation curve. Similar investigation in Table 2 displays a series of results for percentage saturation of myoglobin and partial pressure of oxygen. Figure 3 is produced by assigning random values to free parameters in description of $C^{1}$ rational trigonometric cubic function (1) which fails to conserve the monotone trend of data. Algorithm 2 developed in Section 4 is applied to remove this drawback and Figure 4 displays the required result. Numerical results corresponding to Figures 2 and 4 are shown in Tables 3 and 4.

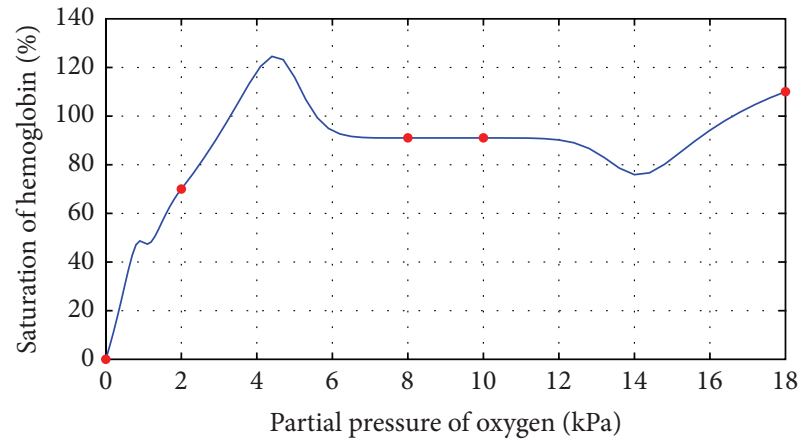

FIGURE 1: $C^{1}$ rational trigonometric cubic function with $\alpha_{i}=1.0$, $\beta_{i}=0.5, \gamma_{i}=1.0, \delta_{i}=2.0$.

The $3 \mathrm{D}$ monotone data set in Tables 5 and 6 are generated from the following functions:

$$
\begin{gathered}
F(x, y)=\sqrt{\frac{x^{2}}{25}+\frac{y^{2}}{16}}, \\
F(x, y)=\log \left(x^{2}+y^{2}\right) .
\end{gathered}
$$

respectively.

Figures 5 and 7 are produced by interpolating the monotone data sets in Tables 5 and 6, respectively, by $C^{1}$ rational 
TABLE 8: Numerical values corresponding to Figure 8.

\begin{tabular}{|c|c|c|c|c|c|c|}
\hline$\left(x_{i}, y_{j}\right)$ & 1 & 2 & 3 & 4 & 5 & 6 \\
\hline \multicolumn{7}{|c|}{ Numerical values of $F_{i, j}^{x}$} \\
\hline 1 & 1.0279 & 0.4623 & 0.2308 & 0.1322 & 0.0843 & 0.0581 \\
\hline 2 & 0.8047 & 0.4778 & 0.2939 & 0.1928 & 0.1341 & 0.0979 \\
\hline 3 & 0.6119 & 0.4581 & 0.3270 & 0.2350 & 0.1731 & 0.1312 \\
\hline 4 & 0.4778 & 0.4012 & 0.3180 & 0.2473 & 0.1928 & 0.1521 \\
\hline 5 & 0.3889 & 0.3466 & 0.2939 & 0.2428 & 0.1987 & 0.1627 \\
\hline 6 & 0.3168 & 0.2966 & 0.2667 & 0.2326 & 0.1991 & 0.1689 \\
\hline \multicolumn{7}{|c|}{ Numerical values of $F_{i, j}^{y}$} \\
\hline 1 & 1.0279 & 0.8047 & 0.6119 & 0.4778 & 0.3889 & 0.3168 \\
\hline 2 & 0.4623 & 0.4778 & 0.4581 & 0.4012 & 0.3466 & 0.2966 \\
\hline 3 & 0.2308 & 0.2939 & 0.3270 & 0.3180 & 0.2939 & 0.2667 \\
\hline 4 & 0.1322 & 0.1928 & 0.2350 & 0.2473 & 0.2428 & 0.2326 \\
\hline 5 & 0.0843 & 0.1341 & 0.1731 & 0.1928 & 0.1987 & 0.1991 \\
\hline 6 & 0.0581 & 0.0979 & 0.1312 & 0.1521 & 0.1627 & 0.1689 \\
\hline \multicolumn{7}{|c|}{ Numerical values of $\beta_{i, j}$} \\
\hline 1 & 13.4612 & 11.8021 & 10.5579 & 9.7618 & 9.2601 & - \\
\hline 2 & 13.9316 & 11.8084 & 10.8374 & 10.3699 & 10.1191 & - \\
\hline 3 & 13.8377 & 12.7622 & 11.9437 & 11.4236 & 11.0979 & - \\
\hline 4 & 13.4933 & 12.9563 & 12.4102 & 11.9764 & 11.6602 & - \\
\hline 5 & 13.2255 & 12.9325 & 12.5819 & 12.2566 & 11.9879 & - \\
\hline 6 & - & - & - & - & - & - \\
\hline \multicolumn{7}{|c|}{ Numerical values of $\gamma_{i, j}$} \\
\hline 1 & 10.5388 & 12.1979 & 13.4421 & 14.2382 & 14.7399 & - \\
\hline 2 & 10.5932 & 11.3237 & 12.0568 & 12.6377 & 13.0617 & - \\
\hline 3 & 10.8043 & 11.1752 & 11.6161 & 12.0237 & 12.3602 & - \\
\hline 4 & 10.9824 & 11.1929 & 11.4696 & 11.7539 & 12.0121 & - \\
\hline 5 & 10.7745 & 11.0675 & 11.4181 & 11.7434 & 12.0121 & - \\
\hline 6 & - & - & - & - & - & - \\
\hline \multicolumn{7}{|c|}{ Numerical values of $\widehat{\beta}_{i, j}$} \\
\hline 1 & 13.4612 & 13.9316 & 13.8377 & 13.4933 & 13.2255 & - \\
\hline 2 & 11.8021 & 11.8084 & 12.7622 & 12.9563 & 12.9325 & - \\
\hline 3 & 10.5579 & 10.8374 & 11.9437 & 12.4102 & 12.5819 & - \\
\hline 4 & 9.7618 & 10.3699 & 11.4236 & 11.9764 & 12.2566 & - \\
\hline 5 & 9.2601 & 10.1191 & 11.0979 & 11.6602 & 11.9879 & - \\
\hline 6 & - & - & - & - & - & - \\
\hline \multicolumn{7}{|c|}{ Numerical values of $\widehat{\gamma}_{i, j}$} \\
\hline 1 & 10.5388 & 10.5932 & 10.8043 & 10.9824 & 10.7745 & - \\
\hline 2 & 12.1979 & 11.3237 & 11.1752 & 11.1929 & 11.0675 & - \\
\hline 3 & 13.4421 & 12.0568 & 11.6161 & 11.4696 & 11.4181 & - \\
\hline 4 & 14.2382 & 12.6377 & 12.0237 & 11.7539 & 11.7434 & - \\
\hline 5 & 14.7399 & 13.0617 & 12.3602 & 12.0121 & 12.0121 & - \\
\hline 6 & - & - & - & - & - & - \\
\hline \multicolumn{7}{|c|}{ Numerical values of $\beta_{i, j+1}$} \\
\hline 1 & 13.7691 & 12.3176 & 11.3888 & 10.8035 & 10.4245 & - \\
\hline 2 & 13.7765 & 12.6436 & 12.0982 & 11.8056 & 11.6334 & - \\
\hline 3 & 14.8893 & 13.9343 & 13.3275 & 12.9476 & 12.7025 & - \\
\hline 4 & 15.1157 & 14.4785 & 13.9724 & 13.6036 & 13.3401 & - \\
\hline 5 & 15.0879 & 14.6788 & 14.2994 & 13.9859 & 13.7398 & - \\
\hline 6 & - & - & - & - & - & - \\
\hline
\end{tabular}


TABLE 8: Continued.

\begin{tabular}{|c|c|c|c|c|c|c|}
\hline$\left(x_{i}, y_{j}\right)$ & 1 & 2 & 3 & 4 & 5 & 6 \\
\hline \multicolumn{7}{|c|}{ Numerical values of $\gamma_{i, j+1}$} \\
\hline 1 & 13.2144 & 14.5622 & 15.4247 & 15.9682 & 16.3201 & - \\
\hline 2 & 12.2673 & 13.0616 & 13.6908 & 14.1502 & 14.4789 & - \\
\hline 3 & 12.1065 & 12.5841 & 13.0257 & 13.3902 & 13.6766 & - \\
\hline 4 & 12.1257 & 12.4254 & 12.7334 & 13.0131 & 13.2509 & - \\
\hline 5 & 11.9898 & 12.3697 & 12.7220 & 13.0131 & 13.2416 & - \\
\hline 6 & - & - & - & - & - & - \\
\hline \multicolumn{7}{|c|}{ Numerical values of $\widehat{\beta}_{i+1, j}$} \\
\hline 1 & 7.0627 & 9.6496 & 12.0876 & 13.2188 & 13.7521 & - \\
\hline 2 & 6.8759 & 8.4746 & 10.6260 & 11.9816 & 12.7945 & - \\
\hline 3 & 7.0547 & 8.2958 & 10.0152 & 11.2619 & 12.1246 & - \\
\hline 4 & 7.2590 & 8.4154 & 9.8191 & 10.8928 & 11.7015 & - \\
\hline 5 & 7.4425 & 8.6142 & 9.8100 & 10.7305 & 11.4556 & - \\
\hline 6 & - & - & - & - & - & - \\
\hline \multicolumn{7}{|c|}{ Numerical values of $\widehat{\gamma}_{i+1, j}$} \\
\hline 1 & 13.2144 & 12.2673 & 12.1065 & 12.1257 & 11.9898 & - \\
\hline 2 & 14.5622 & 13.0616 & 12.5841 & 12.4254 & 12.3697 & - \\
\hline 3 & 15.4247 & 13.6908 & 13.0257 & 12.7334 & 12.7220 & - \\
\hline 4 & 15.9682 & 14.1502 & 13.3902 & 13.0131 & 13.0131 & - \\
\hline 5 & 16.3201 & 14.4789 & 13.6766 & 13.2509 & 13.2416 & - \\
\hline 6 & - & - & - & - & - & - \\
\hline
\end{tabular}

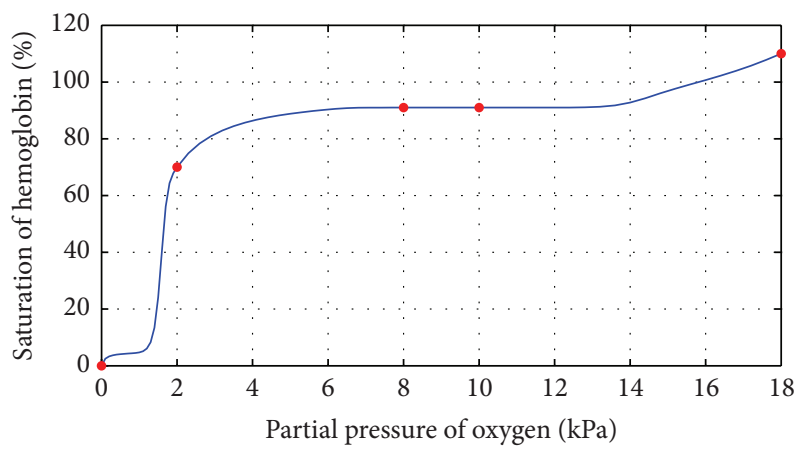

FIGURE 2: $C^{1}$ monotone rational trigonometric cubic function with $\alpha_{i}=2.6, \delta_{i}=0.4$.

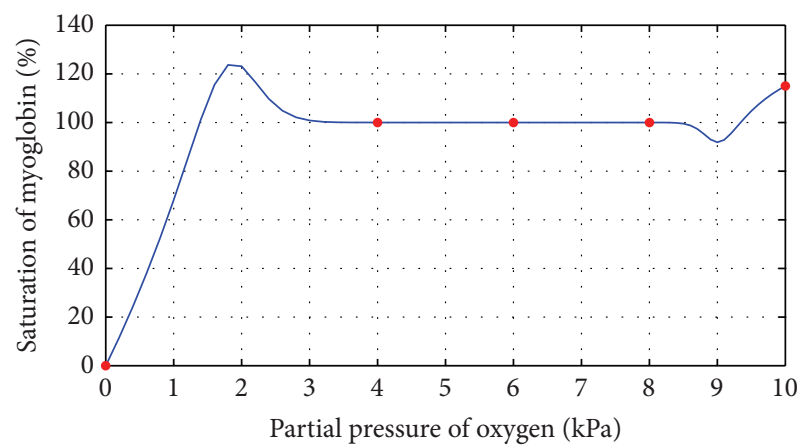

FIgURE 3: $C^{1}$ rational trigonometric cubic function with $\alpha_{i}=$ 2.5, $\beta_{i}=0.5, \gamma_{i}=0.5, \delta_{i}=2.0$.

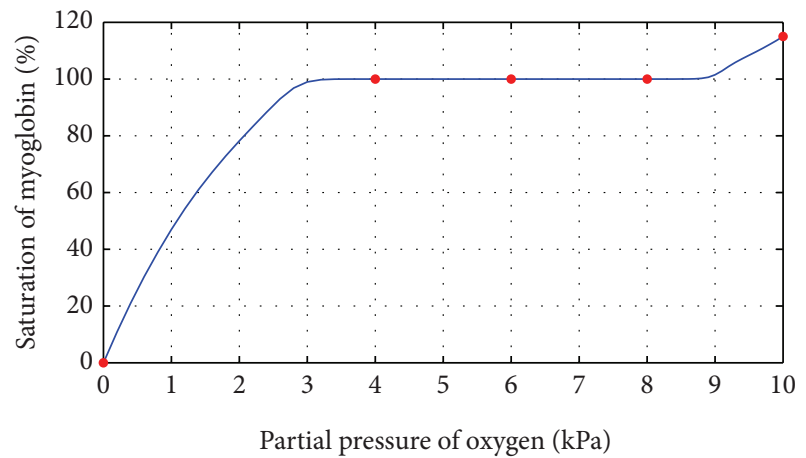

FIGURE 4: $C^{1}$ monotone rational trigonometric cubic function with $\alpha_{i}=2.0, \delta_{i}=0.5$.

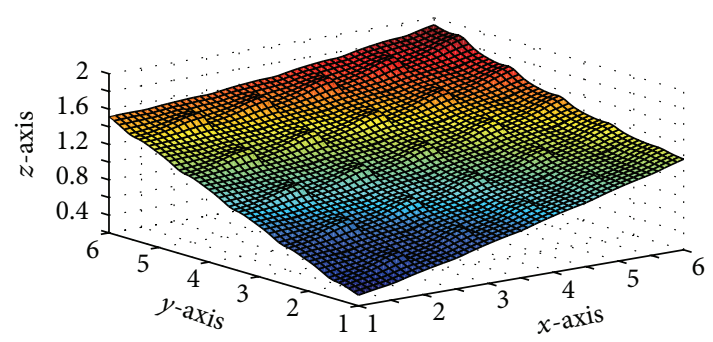

FIGURE 5: $C^{1}$ rational trigonometric bicubic function with $\alpha_{i, j}=14$, $\beta_{i, j}=15, \gamma_{i, j}=6, \delta_{i, j}=7, \alpha_{i, j+1}=8, \beta_{i, j+1}=8, \gamma_{i, j+1}=4, \delta_{i, j+1}=$ $9, \widehat{\alpha}_{i, j}=6, \widehat{\beta}_{i, j}=5, \widehat{\gamma}_{i, j}=4, \widehat{\delta}_{i, j}=8, \widehat{\alpha}_{i+1, j}=15, \widehat{\beta}_{i+1, j}=2, \widehat{\gamma}_{i+1, j}=$ $12, \widehat{\delta}_{i+1, j}=8$. 


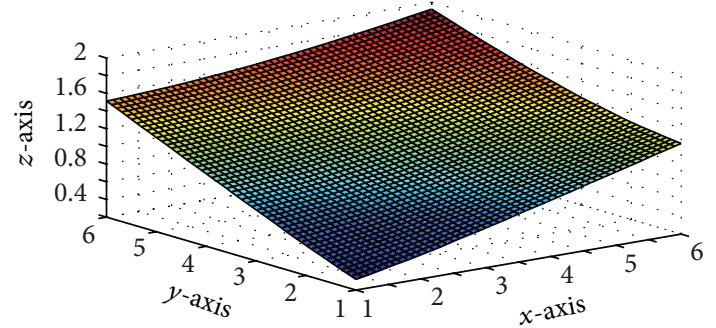

FIGURE 6: $C^{1}$ monotone rational trigonometric bicubic function with $\alpha_{i, j}=12, \delta_{i, j}=12, \alpha_{i, j+1}=14, \delta_{i, j+1}=13, \widehat{\alpha}_{i, j}=12, \widehat{\delta}_{i, j}=$ $12, \widehat{\alpha}_{i+1, j}=15, \widehat{\delta}_{i+1, j}=13$.

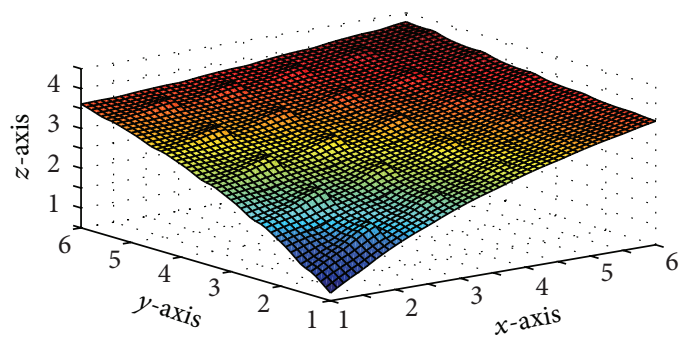

Figure 7: $C^{1}$ rational trigonometric bicubic function with $\alpha_{i, j}=$ $14, \beta_{i, j}=8, \gamma_{i, j}=5, \delta_{i, j}=7, \alpha_{i, j+1}=14, \beta_{i, j+1}=8, \gamma_{i, j+1}=$ $6, \delta_{i, j+1}=13, \widehat{\alpha}_{i, j}=12, \widehat{\beta}_{i, j}=9, \widehat{\gamma}_{i, j}=8, \widehat{\delta}_{i, j}=8, \widehat{\alpha}_{i+1, j}=$ $15, \widehat{\beta}_{i+1, j}=9, \widehat{\gamma}_{i+1, j}=8, \widehat{\delta}_{i+1, j}=8$.

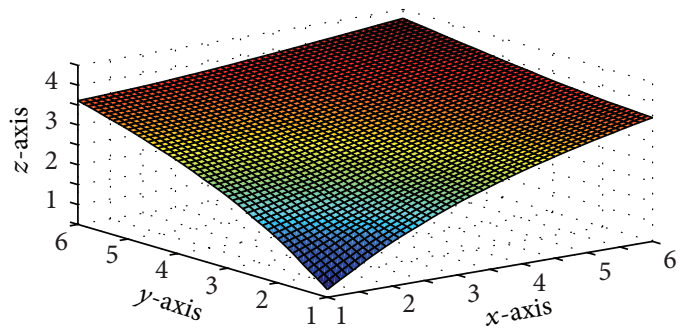

FIGURE 8: $C^{1}$ monotone rational trigonometric bicubic function with $\alpha_{i, j}=12, \delta_{i, j}=12, \alpha_{i, j+1}=13, \delta_{i, j+1}=14, \widehat{\alpha}_{i, j}=10, \widehat{\delta}_{i, j}=$ $10, \widehat{\alpha}_{i+1, j}=15, \widehat{\delta}_{i+1, j}=13$.

trigonometric bicubic function for arbitrary values of free parameter. Monotone surfaces in Figures 6 and 8 are produced by interpolating the same data by the monotonicity preserving scheme developed in Section 5. Tables 7 and 8 enclose numerical results against Figures 6 and 8.

\section{Conclusion}

In this paper, monotonicity of data is retained by developing constraints on free parameters in the specification of rational trigonometric function and bicubic blended function. Authors in $[7,8]$ used algebraic function while the proposed algorithm applies trigonometric function which gives much smoother result due to orthogonality of sine and cosine function. Shape preserving techniques of Butt and Brodlie
[1] required insertion of additional knots. In [12], developed scheme failed to maintain smoothness. The proposed technique is local, affirms smoothness, works well for data with derivatives, and does not require insertion of extra knots. Derivative of trigonometric spline is much lower than that of polynomial spline.

\section{Conflict of Interests}

The authors declare that there is no conflict of interests regarding the publication of this paper.

\section{References}

[1] S. Butt and K. W. Brodlie, "Preserving positivity using piecewise cubic interpolation," Computers \& Graphics, vol. 17, no. 1, pp. 5564, 1993.

[2] P. Costantini and F. Fontanella, "Shape-preserving bivariate interpolation," SIAM Journal on Numerical Analysis, vol. 27, no. 2, pp. 488-506, 1990.

[3] R. J. Cripps and M. Z. Hussain, " $\mathrm{C}^{1}$ monotone cubic Hermite interpolant," Applied Mathematics Letters, vol. 25, no. 8, pp. 11611165, 2012.

[4] R. D. Fuhr and M. Kallay, "Monotone linear rational spline interpolation," Computer Aided Geometric Design, vol. 9, no. 4, pp. 313-319, 1992.

[5] M. S. Floater and J. M. Peña, "Monotonicity preservation on triangles," Mathematics of Computation, vol. 69, no. 232, pp. $1505-1519,2000$.

[6] T. N. T. Goodman, B. H. Ong, and K. Unsworth, "Constrained interpolation using rational cubic splines," in NURBS for Curve and Surface Design, G. Farin, Ed., pp. 59-74, 1991.

[7] M. Z. Hussain and M. Hussain, "Visualization of data preserving monotonicity," Applied Mathematics and Computation, vol. 190, no. 2, pp. 1353-1364, 2007.

[8] M. Z. Hussain and M. Sarfraz, "Monotone piecewise rational cubic interpolation," International Journal of Computer Mathematics, vol. 86, no. 3, pp. 423-430, 2009.

[9] M. Z. Hussain and S. Bashir, "Shape preserving surface data visualization using rational bi-cubic functions," Journal of Numerical Mathematics, vol. 19, no. 4, pp. 267-307, 2011.

[10] M. Z. Hussain, M. Hussain, and A. Waseem, "Shape-preserving trigonometric functions," Computational and Applied Mathematics, 2013.

[11] F. Ibraheem, M. Hussain, M. Z. Hussain, and A. A. Bhatti, "Positive data visualization using trigonometric function," Journal of Applied Mathematics, vol. 2012, Article ID 247120, 19 pages, 2012.

[12] M. Sarfraz, S. Butt, and M. Z. Hussain, "Surfaces for the visualization of scientific data preserving monotonicity," in Proceedings of the 7th IMA Mathematics for Surfaces Conference, T. N. T. Goodman and R. Martin, Eds., pp. 479-495, Dundee, UK, September 1997.

[13] M. Sarfraz, "A rational cubic spline for the visualization of monotonic data: an alternate approach," Computers \& Graphics, vol. 27, no. 1, pp. 107-121, 2003.

[14] M. Sarfraz and M. Z. Hussain, "Data visualization using rational spline interpolation," Journal of Computational and Applied Mathematics, vol. 189, no. 1-2, pp. 513-525, 2006. 
[15] M. Sarfraz, M. Z. Hussain, and M. Hussain, "Shape-preserving curve interpolation," International Journal of Computer Mathematics, vol. 89, no. 1, pp. 35-53, 2012.

[16] M. Sarfraz, M. Z. Hussain, and M. Hussain, "Modeling rational spline for visualization of shaped data," Journal of Numerical Mathematics, vol. 21, no. 1, pp. 63-87, 2013.

[17] L. L. Schumaker, "On shape preserving quadratic spline interpolation," SIAM Journal on Numerical Analysis, vol. 20, no. 4, pp. 854-865, 1983. 


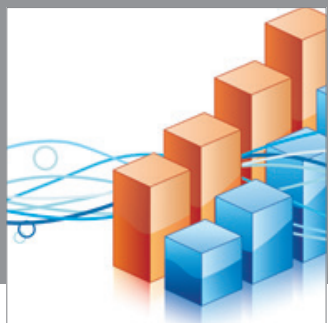

Advances in

Operations Research

mansans

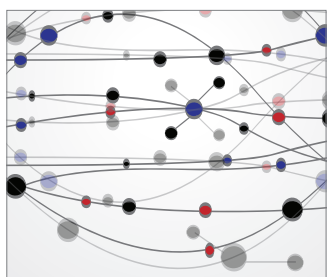

The Scientific World Journal
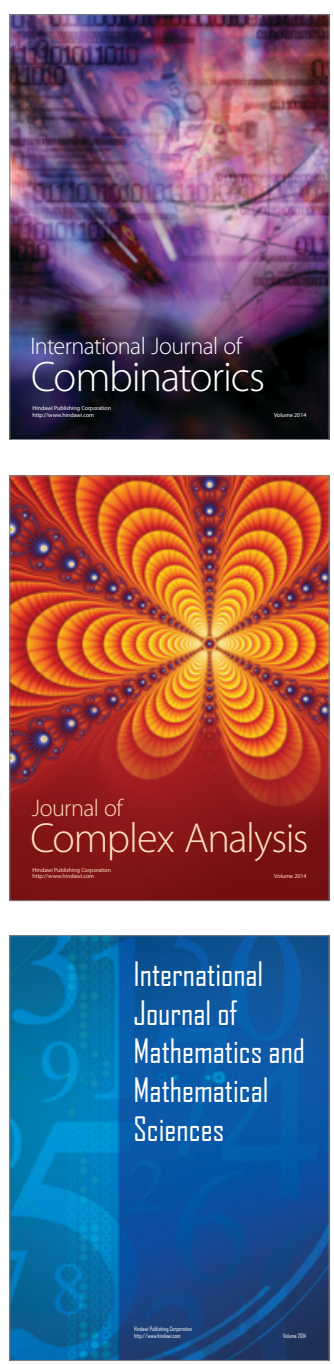
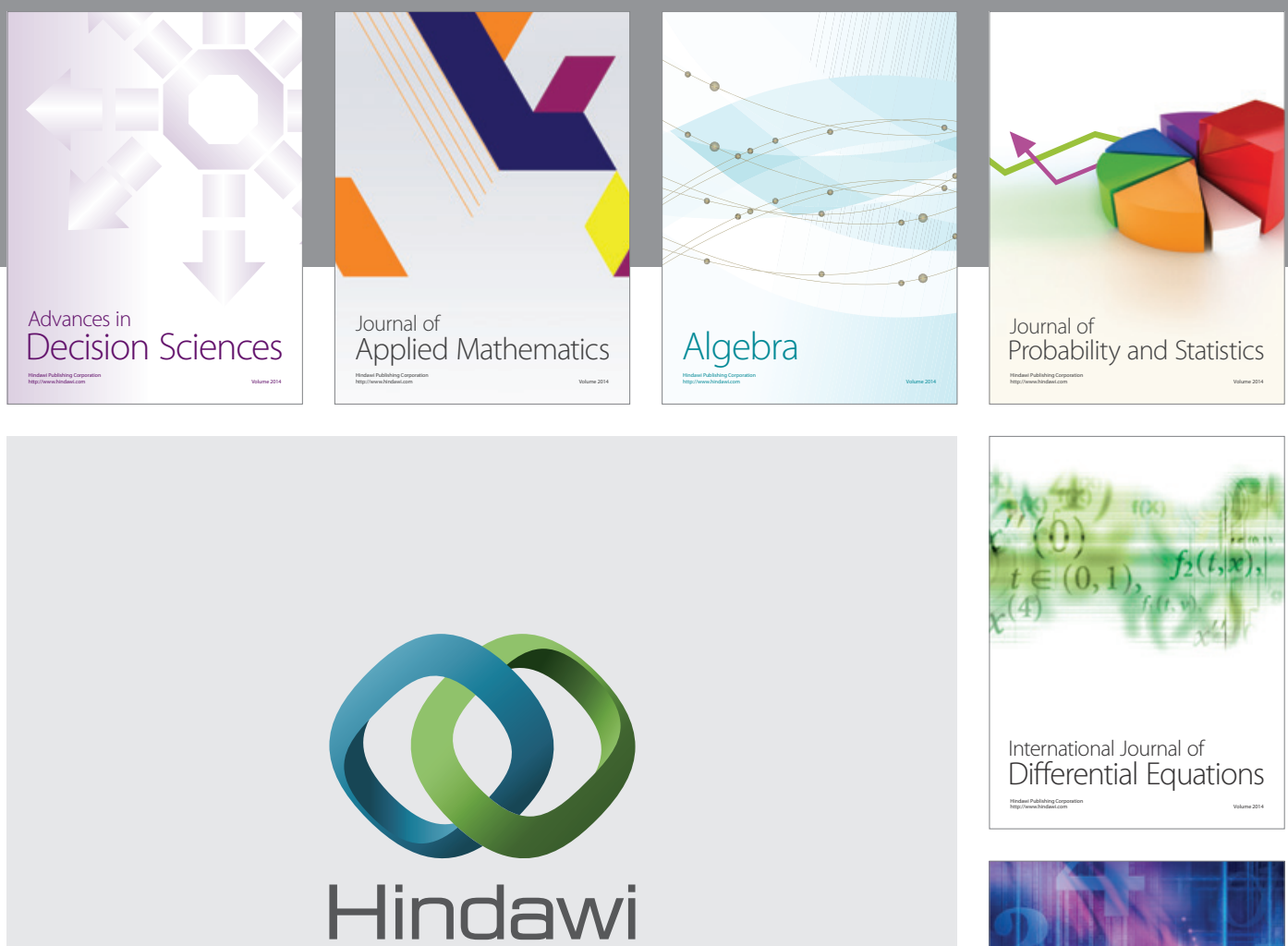

Submit your manuscripts at http://www.hindawi.com
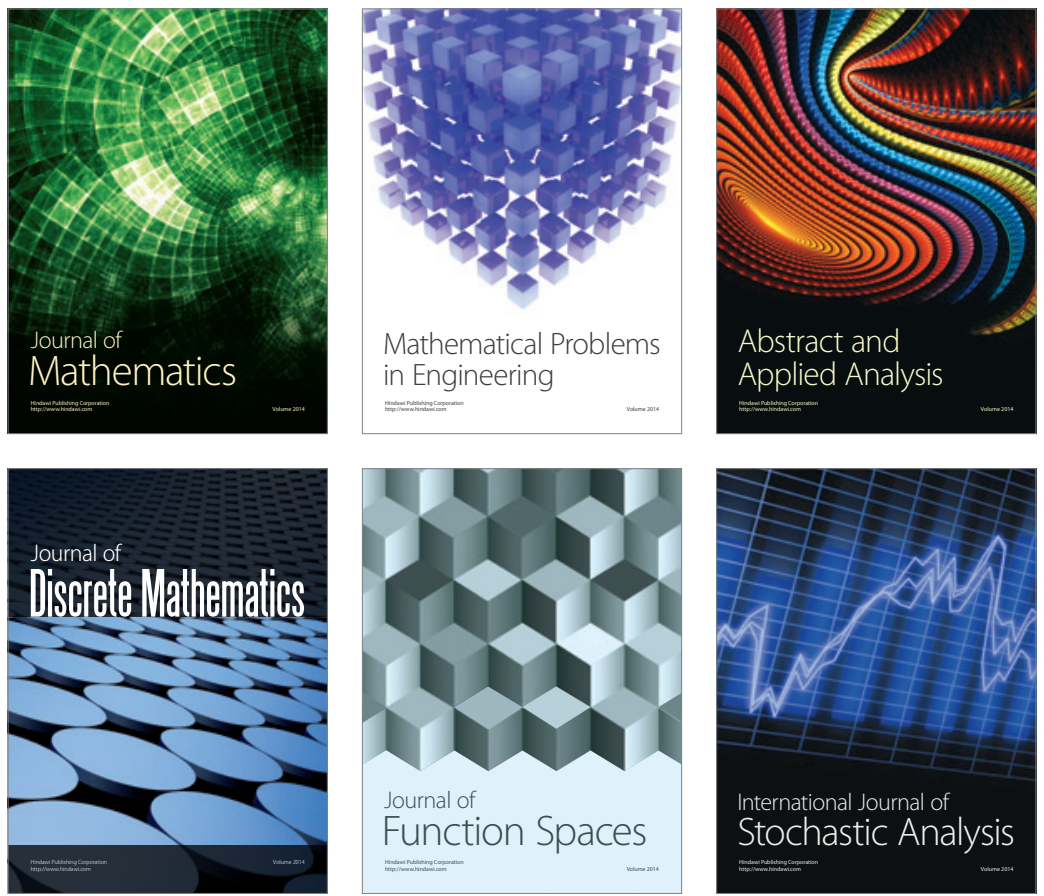

Journal of

Function Spaces

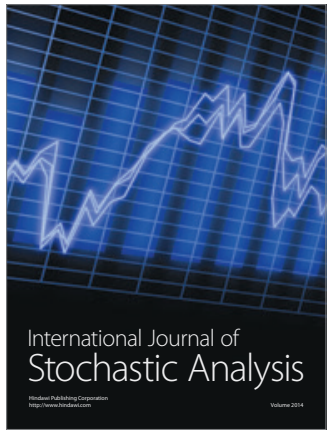

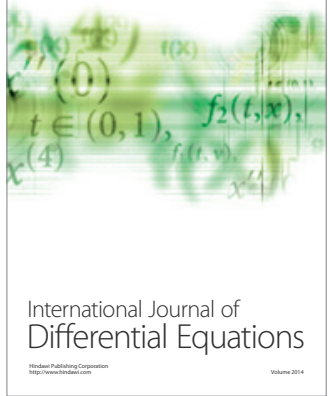
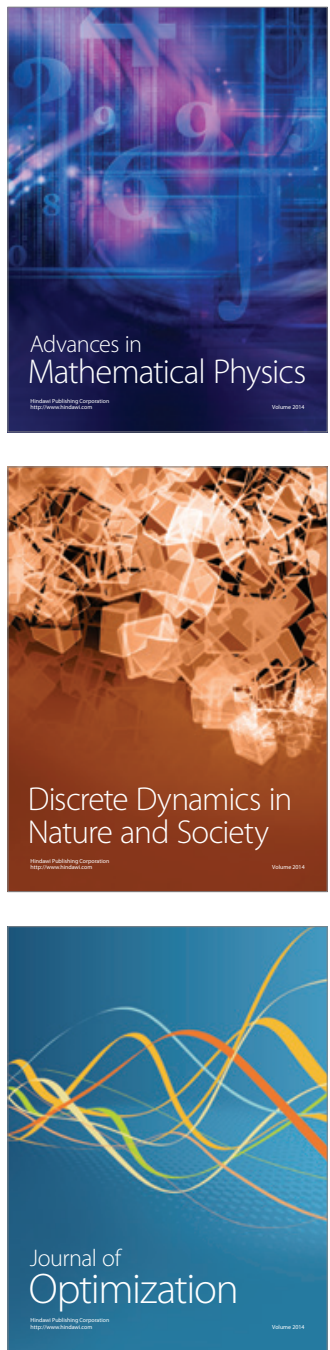